\title{
Hollow Polymer Capsules for Drug Delivery
}

\author{
Yuancheng $\mathrm{Ji}^{a}$ and Junqiu Liu ${ }^{*, a, b}$ \\ a State Key Laboratory of Supramolecular Structure and Materials, College of Chemistry, Jilin University, \\ 2699 Qianjin Street, Changchun, Jilin 130012, China \\ ${ }^{b}$ College of Material, Chemistry and Chemical Engineering, Hangzhou Normal University, Hangzhou,
} Zhejiang 311121 China

Email: junqiuliu@jlu.edu.cn (J. L.)

\begin{abstract}
Hollow polymer capsules (HPC) are one of the widely studied topics in two-dimensional (2D) materials as artificial cargo carriers that simulate natural cells containing life substances and complex life activities, In the past two decades, methodologies and application scenarios for the synthesis of polymer capsules with diversified morphology and multiple functions have received extensive attention. In particular, well-defined HPC with hollow compartments can serve as a drug delivery system with high load capacity, low toxicity, targeted and efficient release. This perspective aims to briefly summarize the developed fabrication strategy of HPC, evaluate their application characteristics in drug encapsulation and delivery, and propose a development direction of HPC as an outstanding drug carrier system.
\end{abstract}

Keywords hollow polymer capsules, drug delivery, methodology, 2D materials

Two-dimensional (2D) materials have been the subject of many classic studies in transdisciplinary research. During the last decade, the link between artificial materials and life science has been at the center of much attention. Notably, the construction of hollow polymer capsules (HPC) as artificial cells for bioactive substance carriers and microreactors is one of the critical advances in bio-nanomedicine. ${ }^{[1]}$ Since Caruso and his co-workers firstly reported hollow inorganic silicon and inorganic-polymer hybrid spheres through the electrostatic layer-by-layer (LBL) self- assembly on colloidal templates, ${ }^{[2]}$ HPC methodological research and functionalization application exploration, represented by the template method, has attracted widespread attention from researchers. The drug delivery system adjusts the pharmaceutical properties or promotes the biological adaptation, thereby regulating the temporal and spatial distribution of the drug in the body. It is important to note that pharmacologically active agents are not inherently effective, and their effectiveness depends directly on how they are manipulated. ${ }^{[3]}$ Therefore, by rationally designing synthetic pathways for hollow polymer capsules (Figure 1), we can endow them with a variety of functions and prepare intelligent drug carriers to achieve controlled delivery and release under various stimuli. Herein, we attempt to briefly categorize the synthesis methodology, thereby pointing to emerging preparative ideas and the prospects for HPC in drug delivery.

\section{Traditional Fabrication Methods}

HPC is usually defined by a nanometer/micrometer-scale system with a well-defined shell structure and a large blank cavity, which imparts the spatial characteristics of a large surface area, low density, and the ability to envelop goods. Considering the differences in various physicochemical and material properties, it is usually common to classify the HPC preparation into two main categories: templated and non-templated methods. ${ }^{[4]}$ The template method adopts the bottom-up strategies and performs post-treatment processes such as deposition, coating, cross-linking, and other means of target materials on

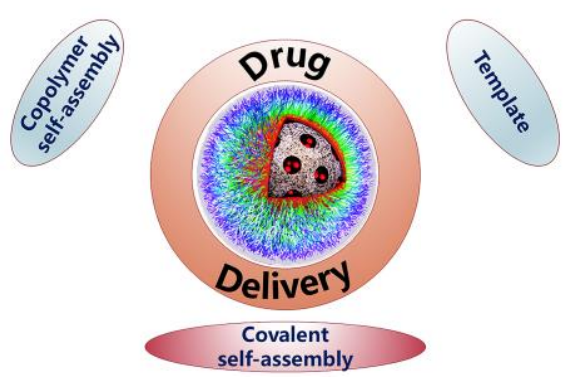

Figure 1 Conventional methods and newly ones of producing HPC for drug delivery.

spherical-like substrates or precursors such as microemulsions and micelles with deformable properties. The internal template is removed through etching and dissolution, and finally, we can obtain a nano/micro-assembly with a cavity structure.

The hard template method generally employs solid particles with stereotyped texture as the substrate, so the internal spatial characteristics of the hollow capsule as well as the configuration and functionalization potential of the shell structure is mainly determined by the key parameters: template volume, surface reactive active components, and microporous size distribution. Brigitte et al. ${ }^{[5]}$ reported a rapid one-pot synthesis of ABA-type triblock copolymer brushes on silica template via surface-initiated single electron transfer living radical polymerization (SI-SET-LRP). Notably, compared to the traditional surface-initiated living radical polymerization (SI-LRP), which requires a tedious purification process, copolymer polymer brushes can be prepared in $15 \mathrm{~min}$ on the surface of silica to obtain HPC by photocrosslinking and etching of the silica in a fast and effective way. It is exciting to highlight that this new hollow nanocarrier features a permeability switch, where temperature plays a significant role and further smart half-openfull-open properties in $\mathrm{pH}$ response. Researchers probe the permeability changes in composite vesicles in response to 


\section{Perspective}

temperature and $\mathrm{pH}$ through dual enzyme cascade reactions inside and outside the capsule. The results show that the valve-like tunable permeability of this hollow nanoreactor provides a novel platform for efficient drug envelopment, stable drug delivery, and intelligent, responsive drug release.

Although the hard template method can provide a simple way to fabricate a hollow particle without too much-thinking complexity, it is somewhat complicated and corrosive due to the use of mordant reagents $\left(\mathrm{HF}, \mathrm{HCl}, \mathrm{HNO}_{3}, \mathrm{NaOH}\right)$ during the etching processes. Amphiphilic molecules may be capable of self-assembling into different micelles or vesicle structures when concentrations in solution exceed a critical micelle concentration, and such micelles and vesicles may serve as soft templates to guide the formation of hollow structures. Classification according to template type, soft template methods can be subdivided into (1) emulsion template method, (2) vesicle/ micelle template method, (3) gas bubble template method. In contrast, the soft template method enables a more complex hierarchical structure to be obtained by eliminating the complex template removal process and enveloping the drug in situ during capsule formation. ${ }^{[6]}$ These techniques have been widely developed and applied in the construction of various functional 2D hollow particles.

The self-templating method is a relatively novel concept, which refers to the procedure of synthesizing micro- and nano-precursors before transforming them into hollow structures. Here, the precursors not only play a supporting role but are also directly involved in the formation of the shell layer of HPC. Wang et al. ${ }^{[7]}$ reported a new nanomedical platform successfully prepared by a hollow-excavated strategy and a surface protection etching based self-templating method. Inside this composite nanostructure, a lamellar and porous silica shell is encased by a gold nanocage sealed with $p$-aminothiophenol (pATP), with a functionalized cell-transmembrane peptide Tat on the exterior wall. Due to the rational design, the nanomedical platform can be used as a hollow carrier to encapsulate and deliver pharmaceutical drugs efficiently. Secondly, the gold nanocage with electromagnetic enhanced properties can be used as a substrate for surface-enhanced Raman scattering (SERS) to track the internal engulfment process of MCF-7 breast neoplastic cells to the nanotherapeutic system. At the same time, the gold nanocage with strong infrared absorption properties also endows the platform with a photothermal therapy feature.

In contrast to the bottom-up construction mindset of the template method, block copolymers undergo a self-assembly behavior based on multiple weak interactions in a specific solvent through differences in the interaction of different parts with the solvent, which leads to the direct construction of hollow polymer capsules. ${ }^{[8]}$ Important to note is that solvent interacting with block copolymers in biopharmaceuticals, such as drug delivery, is strictly limited compared to other construction methods, where strict aqueous systems restrict the assembly of complex hollow structures to some extent.

\section{Defects and Development of Traditional Methods in Drug Delivery}

Until now, the fabrication of HPC based on the template method and block copolymer self-assembly has been reported extensively, so that the research system has evolved from simple monolayer nanocomposites to polymer capsules with intricate morphology and function, which have been applied in drug encapsulation and delivery. However, for the development of biopharmaceutical systems, the hard template method requires the general use of toxic solvents or etching agents to remove the template, which would inevitably leave traces of toxic agents. Even though the soft template method avoids the tedious process of template removal, the coating procedure generally involves additional surface modification steps, which will limit the yield of hollow capsules and increase the cost price. Also, the polydispersity of the material prepared by the soft template method makes it challenging to obtain hollow structures of uniform size and monodispersity. The self-templating method simplifies the fabrication steps and promises large-scale production due to reduced material costs, but the singularity of the template material dramatically limits the application of polymeric capsules. For block copolymer self-assembly methods, the requirements of precise molecular composition design, lower concentration of precursors, and subsequent necessary steps such as shell cross-linking, also pose challenges for preparations of drug carriers on a massive scale. $^{[6]}$

Besides, the polymer capsules are required to fulfill several vital criteria as an ideal drug delivery system for enhancing drug efficacy and reducing side effects: excellent biocompatibility, efficient and straightforward preparation methods, the accessible introduction of functionalized groups for targeted transport, and controlled release at designated sites. ${ }^{[9-11]}$ There is clinical significance in terms of how these necessary conditions are integrated into a hollow polymer capsule. Unfortunately, the above-mentioned developed construction methods have not been able to scale up the construction of medical polymer capsules with practical requirements.

\section{Covalent Self-Assembly Polymer Capsule}

Considering the above, the development of new polymeric capsules based on covalent chemistry has attracted the attention of the scientific community. The covalent self-assembly mainly depends on the equilibrium of system free energy and entropy to be the most stable situation, as well as the noncovalent methods, corresponding with the "self-checking" and "self-error-correcting" processes under thermodynamic control. Notably, the key to accomplishing the covalent assembly is to introduce some active points at the periphery of constructional blocks to provide a growing flat oligomer from which the $2 \mathrm{D}$ materials with diversified morphology present eventually.

The difference between noncovalent and covalent methods lies in that it is some multi-diversified patches that play a critical role after initially partial polymerization, like the traditional assembly of small molecules, to cross-link and aggregate by forming covalent bonds with some influence of solvent effect, bending rigidity of the system, and orientation of the reactive groups. ${ }^{[12]}$

Whereas very few covalent self-assembly methods are reported to achieve fast, mass-preparable, easily functionalized polymer capsules.

In 2007, Kim et al. ${ }^{[13]}$ firstly reported a template-free construction of covalent self-assembled polymer vesicles using cucurbit[6]uril (CB[6]) as a building block, employing a rigid structure with planar lateral active reaction sites, and a flexible linker of appropriate length. Theoretical calculations suggest that the final morphology of the assembly system will be effectively regulated by tuning the solvent type of the assembly system, the bending stiffness of the system, and the orientation of the active group.

Recently, Liu et al. ${ }^{[14]}$ developed a new idea to fabricate 2D nanomaterials based on orthogonal dynamic covalent self-assembly by preparing symmetrical building blocks with boroxane connections using 4-formylphenylboronic acid symmetrical connections and performing Schiff base reactions between flexible linkers with dual amino ends and building blocks (Figure 2). Through the dynamic chemistry of Schiff 


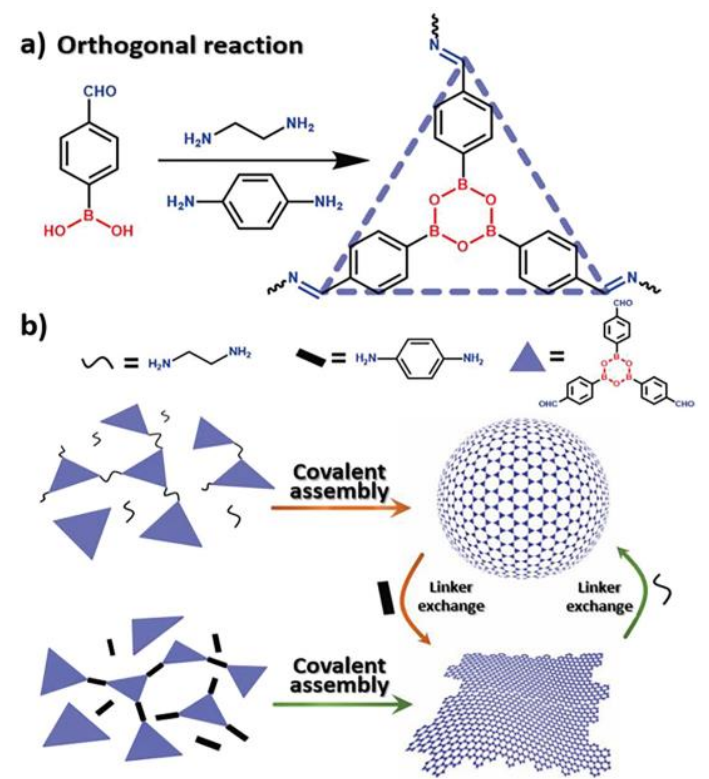

Figure 2 a) Scheme of the orthogonal reactions; b) the structure of two different kinds of linkers. Reprinted with permission from Ref. [14] Copyright (2020) John Wiley and Sons.

base, a kinetic transformation of the hollow nanocapsule/ nanosheet under different diaminoterminal linker cross-linking was achieved. Depending on the covalent chemistry for bonding stability and the wide variety of reactions, we can significantly enrich the selection of prepared materials and add more different intelligent responsiveness features than other methods to produce $2 \mathrm{D}$ nanomaterials.

The first covalent polymer capsule with hypoxic response has been reported recently by Wang et al. ${ }^{[15]}$ Successful fabrication of covalent vesicles was achieved by introducing an azobenzene molecule characterized by reducible fracture under anaerobic conditions, which served as a linker with perhydroxycucurbit[6]uril as the building block. Remarkably, a tumor microenvironment (TME) with high levels of hypoxia due to the accumulation of necrotic tissue at the tumor site allows for efficient release of covalent drug-carrier vesicles at the disease site in response to hypoxia, thus extending the intelligent features of covalent self-assembled drug delivery systems.

\section{Conclusions and Outlook}

Hollow polymer capsules are widely applied in catalysis, sensing, biomedical and other fields because of their outstanding physicochemical properties, requiring further development in the future. Various methodologies for the preparation of hollow polymer capsules have certain advantages such as batch preparation, low cost, intelligent efficiency, and excellent biocompatibility. Particularly in drug delivery applications, many polymer capsules have been industrialized as drug carriers. Therefore, pursuing the development of methodologies for the construction of polymer capsules, especially the fusion and breakthrough of various methods based on the existing ones, to produce a drug carrier system that is functionally diverse, highly stable and efficient, and quickly prepared to target the complicated life systems, which may be the further direction of progress.

\section{Acknowledgement}

This work was supported by the National Key R\&D Program of China (Grant No. 2018YFA0901600).

\section{Conflict of Interest}

The authors declare no conflict of interest.

Copyright ( $) 2021$ Yuancheng Ji and Junqiu Liu. This article is an open access article distributed under the terms and conditions of the Creative Commons Attribution (CC BY) license (http://creativecommons.org/ licenses/by/4.0/). The use, distribution or reproduction in other forums is permitted, provided the original author(s) or licensor are credited and that the original publication in this journal is cited, in accordance with accepted academic practice. No use, distribution or reproduction is permitted which does not comply with these terms.

\section{References}

[1] Xu, C.; Hu, S.; Chen, X. Artificial Cells: From Basic Science To Applications. Mater Today 2016, 19, 516-532.

[2] Caruso, F.; Caruso, R. A.; Möhwald, H. Nanoengineering Of Inorganic And Hybrid Hollow Spheres By Colloidal Templating. Science 1998, 282, 1111-1114.

[3] Tibbitt, M. W.; Dahlman, J. E.; Langer, R. Emerging Frontiers In Drug Delivery. J. Am. Chem. Soc. 2016, 138, 704-717

[4] Wang, X.; Feng, J.; Bai, Y.; Zhang, Q.; Yin, Y. Synthesis, Properties, And Applications Of Hollow Micro-/Nanostructures. Chem. Rev. 2016, 116, 10983-11060.

[5] Liu, X.; Appelhans, D.; Voit, B. Hollow Capsules With Multiresponsive Valves For Controlled Enzymatic Reactions. J. Am. Chem. Soc. 2018, 140, 16106-16114.

[6] Ramli, R. A. Hollow Polymer Particles: A Review. RSC Adv. 2017, 7, 52632-52650.

[7] Hu, F.; Zhang, Y.; Chen, G.; Li, C.; Wang, Q. Double-Walled Au Nanocage/SiO2 Nanorattles: Integrating SERS Imaging, Drug Delivery And Photothermal Therapy. Small 2015, 11, 985-993.

[8] Larrañaga, A.; Lomora, M.; Sarasua, J. R.; Palivan, C. G.; Pandit, A. Polymer Capsules As Micro-/Nanoreactors For Therapeutic Applications: Current Strategies To Control Membrane Permeability. Prog. Mater. Sci. 2017, 90, 325-357.

[9] Zhao, L.; Xiao, C.; Wang, L.; Gai, G.; Ding, J. Glucose-Sensitive Polymer Nanoparticles For Self-Regulated Drug Delivery. Chem. Commun. 2016, 52, 7633-7652.

[10] Marturano, V.; Cerruti, P.; Giamberini, M.; Tylkowski, B.; Ambrogi, V. Light-Responsive Polymer Micro-And Nano-Capsules. Polymers (Basel) 2017, 9.

[11] Thambi, T.; Park, J. H.; Lee, D. S. Hypoxia-Responsive Nanocarriers For Cancer Imaging And Therapy: Recent Approaches And Future Perspectives. Chem. Commun. 2016, 52, 8492-8500.

[12] Baek, K.; Hwang, I.; Roy, I.; Shetty, D.; Kim, K. Self-Assembly Of Nanostructured Materials Through Irreversible Covalent Bond Formation. Acc. Chem. Res. 2015, 48, 2221-2229.

[13] Kim, D.; Kim, E.; Kim, J.; Park, K. M.; Baek, K.; Jung, M.; Ko, Y. H.; Sung, W.; Kim, H. S.; Suh, J. H.; Park, C. G.; Na, O. S.; Lee, D. K.; Lee, K. E.; Han, S. S.; Kim, K. Direct Synthesis Of Polymer Nanocapsules With A Noncovalently Tailorable Surface. Angew. Chem. Int. Ed. 2007, 46, 3471-3474.

[14] Tian, R.; Fan, X.; Liu, S.; Li, F.; Yang, F. H.; Li, Y. J.; Luo, Q.; Hou, C. X.; Xu, J. Y.; Liu, J. Q. Morphological Transformation Between Orthogonal Dynamic Covalent Self-Assembly Of Imine-Boroxine Hybrid Polymer Nanocapsules And Thin Films via Linker Exchange. Macromol. Rapid Commun. 2020, 41, 1900586.

[15] Sun, C.; Yue, L.; Cheng, Q.; Wang, Z.; Wang, R. MacrocycleBased Polymer Nanocapsules For Hypoxia-Responsive Payload Delivery. ACS Mater. Lett. 2020, 2, 266-271. 